\title{
LINK BETWEEN PAY FOR PERFORMANCE INCENTIVES AND PHYSICIAN PAYMENT MECHANISMS: EVIDENCE FROM THE DIABETES MANAGEMENT INCENTIVE IN ONTARIO ${ }^{1}$
}

\author{
JASMIN KANTAREVIC ${ }^{\mathrm{a}, \mathrm{b}, \mathrm{c}, *}$ and BORIS KRALJ ${ }^{\mathrm{a}}$

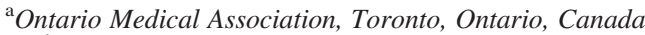 \\ ${ }^{\mathrm{b}}$ University of Toronto, Toronto, Ontario, Canada \\ ${ }^{\mathrm{c}}$ Institute for Labor Studies, Bonn, Germany
}

\begin{abstract}
Pay for performance (P4P) incentives for physicians are generally designed as additional payments that can be paired with any existing payment mechanism such as a salary, fee-for-services and capitation. However, the link between the physician response to performance incentives and the existing payment mechanisms is still not well understood. In this article, we study this link using the recent primary care physician payment reform in Ontario as a natural experiment and the Diabetes Management Incentive as a case study. Using a comprehensive administrative data strategy and a difference-in-differences matching strategy, we find that physicians in a blended capitation model are more responsive to the Diabetes Management Incentive than physicians in an enhanced fee-for-service model. We show that this result implies that the optimal size of P4P incentives vary negatively with the degree of supply-side cost-sharing. These results have important implications for the design of P4P programs and the cost of their implementation. Copyright ( 2012 John Wiley \& Sons, Ltd.
\end{abstract}

Received 15 May 2012; Revised 6 September 2012; Accepted 2 November 2012

KEY WORDS: pay for performance; physician remuneration; diabetes management

JEL Classification: I10; I12; I18

\section{INTRODUCTION}

Pay for performance (P4P) programs have become increasingly popular in recent health care reforms. Two well-known examples include the Quality and Outcomes Framework in the UK and the California Pay for Performance Program in the USA, but there are similar programs in many other countries. ${ }^{2}$ The $\mathrm{P} 4 \mathrm{P}$ programs provide incentives to health care providers for achieving selected performance targets, such as improving preventive and chronic care, patient experience and the use of information technology. The broad goal of these programs is to enhance health care quality, which is expected to improve long-term patients' health and reduce health care costs. ${ }^{3}$ Such promising goals put the P4P programs at the front and centre of many recent health care reforms.

*Correpondence to: Ontario Medical Association, 150 Bloor Street West, Suite 900, Toronto, Ontario, Canada M5S 3C1. E-mail: jasmin. kantarevic@oma.org

${ }^{1}$ The views expressed in this article are strictly those of the authors. No official endorsement by the Ontario Medical Association is intended or should be inferred.

${ }^{2}$ For an overview of these programs, see for example Smith and York (2004) for the UK, the Integrated Healthcare Association (2006) for California, and references in Frolich et al. (2007) for other countries.

${ }^{3}$ See, for example, Dusheiko et al. (2011) for the effect of the Quality and Outcomes Framework on reducing hospital costs and mortality. 
Changing physician practice is a critical step for implementing successful P4P programs. However, recent empirical evidence on the effect of $\mathrm{P} 4 \mathrm{P}$ programs on physician practice is quite mixed. ${ }^{4}$ This puzzling result can be explained in at least two ways. First, there are significant differences across studies in the type of evaluation methodology used to identify the P4P effect, such as the sample size, the nature of comparison group and the set of included confounding factors. Second, there is wide variation in the structure of P4P programs, such as the size of financial incentives, the use of absolute versus relative targets and the use of individualbased versus group-based payments. Consequently, it is not clear whether the lack of consensus in the literature on the effect of $\mathrm{P} 4 \mathrm{P}$ programs is due to methodological shortcomings or because some $\mathrm{P} 4 \mathrm{P}$ programs are just poorly designed. In this study, we focus on the second question of the optimal design of P4P programs. Compared with the literature on whether availability of a specific P4P program affects physician behaviour, the empirical evidence on this question is still quite limited, which reduces our ability to design and implement successful P4P programs. ${ }^{5}$

We contribute to this literature by examining how the optimal size of P4P incentives depends on the supplyside cost-sharing in the physician compensation mechanisms. This cost-sharing refers to the degree to which physicians are reimbursed for incremental services, after receiving any fixed payment. The two extreme examples of cost-sharing are the fee-for-service model, with no cost-sharing, in which physicians receive the full value of incremental services but no fixed payment, and the pure capitation model, with full cost-sharing, in which physicians receive a fixed payment per patient but no reimbursement for incremental services. This question is of policy interest in many countries in which physicians practice in models with various degrees of cost-sharing, such as in Canada and in the USA, where policy makers have to determine the size of P4P incentives. The question is also relevant in countries with a single predominant type of physician compensation mechanism, such as in the UK, where the introduction of new P4P programs may be contemplated along with changes in the degree of supply-side cost-sharing.

In Section 2, we show that the relationship between the optimal size of $\mathrm{P} 4 \mathrm{P}$ incentives and the supply-side cost-sharing depends critically on the link between the physician response to the P4P programs and the type of physician compensation mechanism. We study this link empirically using the recent primary care reform in Ontario as a natural experiment. In this reform, new compensation models with varying degrees of supply-side cost-sharing were sequentially introduced. We use the differential timing of the introduction of these models and the physician transition between the models as a main source of identification. Specifically, we study the physician response to the Diabetes Management Incentive (DMI), a C\$60 per patient annual bonus that physicians receive for planned, ongoing management of diabetic patients according to elements required by the Canadian Diabetes Association Clinical Practice Guidelines, such as tracking and monitoring of HbA1C, health promotion counselling and patient self-management support. We compare this response between physicians practicing in an enhanced fee-for-service model (the Family Health Groups; FHG) and a blended capitation model (the Family Health Organizations; FHO). These two models are currently the most prevalent payment models in Ontario, comprising approximately two-thirds of all primary care physicians. We provide more institutional background on these two models and on the DMI in Section 3.

Participation of physicians in the new payment models is voluntary, which generates concerns about the selection bias if, as expected, factors that affect physician participation in a model also affect their response to the DMI. To address this problem, we use a difference-in-difference matching strategy, which allows us to control for unobserved, time-invariant physician heterogeneity. This empirical strategy is discussed in detail in Section 4. In addition, the matching approach is particularly appealing in our study because of the availability of rich administrative data, described in Section 5, which includes medical profiles of almost all physicians in Ontario that can be used to predict the physician choice of the compensation model. Our focus

\footnotetext{
${ }^{4}$ For recent surveys, see for example Armour et al. (2001), Christianson et al. (2008), Li et al. (2011), Petersen et al. (2006), Town et al. (2005), and Rosenthal and Frank (2006).

${ }^{5}$ For a recent review, see Frolich et al. (2007).
} 
on Ontario is also attractive because it is a single payer system with universal health insurance coverage. Therefore, all physicians within a compensation model face the same financial incentives and demand for medical services is unlikely to be affected by changes in the incentives offered to physicians.

Our results, presented in Section 6, indicate that physicians in the blended capitation model are approximately $12 \%$ more likely to participate in the DMI than physicians in the enhanced fee-for-service model. We also find that diabetic patients enrolled to the capitation physicians are approximately $8 \%$ more likely to receive the DMI services than diabetic patients enrolled to the fee-for-service physicians. These results suggest that the physician response to the P4P incentives varies positively with the degree of supply-side costsharing. Furthermore, these results imply that, for a given compensation mechanism, the optimal size of the $\mathrm{P} 4 \mathrm{P}$ incentives varies negatively with the degree of cost-sharing. Additional comments and our conclusions are presented in Section 7.

Our analysis contributes to the existing literature in three main ways. First, as mentioned, understanding the link between P4P programs and physician payment mechanisms has important implications for both the design of effective P4P programs and the cost of their implementation. Second, diabetes is one of the most common and costly of all chronic diseases. ${ }^{6}$ In addition, it is relatively well understood medically, and there is broadbased agreement on how to manage the disease. Despite this professional knowledge, however, there is widespread concern that diabetes is poorly managed and that it can be significantly improved through incentive programs. Lastly, understanding the effect of different payment models on quality of patient care has been an important policy question for a long time. ${ }^{7}$ Most of the earlier literature focused on cases in which quality could not be observed or verified. Relatively less is known about the effect of payment models when verifiable and contractible indicators of quality are available, such as the DMI in Ontario and many P4P programs in other jurisdictions.

\section{OPTIMAL SIZE OF P4P INCENTIVES AND PHYSICIAN COMPENSATION MECHANISMS}

P4P incentives for physicians are generally designed as additional payments that are paired with the existing physician payment mechanism such as fee-for-service and capitation. In this section, we develop a simple model to reflect this policy problem with the aim of determining the optimal size of a P4P incentive, given the existing payment mechanism. Our model builds on the recent contributions by Eggleston (2005) and Kaarboe and Siciliani (2011). ${ }^{8}$

We assume that a policy maker wishes to maximize the patient benefit $(B)$ net of physician payment $(I)^{9}$ :

$$
W=B-I
$$

The patient benefit depends on the quantity $(q)$ and quality $(e)$ of medical services according to $B(q, e)$, with $B_{q}, B_{e}>0$ and $B_{q q}, B_{e e} \leq 0 .{ }^{10}$ The sign of $B_{q e}$ depends on whether $e$ and $q$ are complements $\left(B_{q e}>0\right)$ or substitutes $\left(B_{q e}<0\right)$ in the patient benefit function.

The physician payment per patient can be represented in a general way as:

\footnotetext{
${ }^{6}$ According to the International Diabetes Federation (2010), the estimated diabetes prevalence for 2010 increased to 285 million, representing $6.4 \%$ of the world's adult population, with a prediction that by 2030 the number of people with diabetes will have increased to 438 million. In Ontario, diabetes costs are estimated at C $\$ 4.9$ billion, or approximately $10 \%$ of the total health care budget (2012 Ontario Budget Speech). Dali et al. (2010) estimate that in the USA, the national economic burden of prediabetes and diabetes reached US $\$ 218$ billion in 2007, with an average annual cost of US $\$ 9677$ for type 2 and US $\$ 14,856$ for type 1.

${ }^{7}$ For recent surveys of this literature, see for example McGuire (2000) and Leger (2008).

${ }^{8}$ Our model can also be interpreted as a special case of the classic multitasking problem, in which both tasks are perfectly observable and the principal cares about the agent's welfare (e.g. Holmstrom and Milgrom, 1991).

${ }^{9}$ This is the same welfare function studied by Kaarboe and Siciliani (2011).

${ }^{10}$ We refer to $q$ as the number of medical services, although it is more properly interpreted as the value of medical services, in which the price per service is normalized to one. Therefore, other prices in the model $(R$ and $r$ ) should be interpreted as relative to the price of medical services.
} 


\section{J. KANTAREVIC AND B. KRALJ}

$$
I=R+r q+p e
$$

where $R$ represents the fixed payment per patient, $r$ represents the reimbursement rate for incremental services and $p$ represents the quality bonus such as the DMI. The degree of supply-side cost-sharing is captured by parameter $r$. With the full cost-sharing, as in the pure capitation model, $R>0$ and $r=0$. With no cost-sharing, as in the pure fee-for-service model, $R=0$ and $r=1$. In a mixed capitation model that is common in many countries, $R>0$ and $r \in(0,1)$.

The policy maker's problem in our environment is to choose $p$, given the existing payment mechanism $(R, r)$. This problem is also subject to two additional types of constraints: the physician participation constraint and the incentive compatibility constraint.

The participation constraint requires that the physician utility from participating in the P4P program is at least as large as that physician's outside option from not participating. Without loss of generality, we normalize this outside option to 0 . The physician utility can be expressed as:

$$
U=\alpha B+I-C(q, e)
$$

where $\alpha \geq 0$ represents the extent of the physician's altruism and $C($.) represents the physician disutility function, with $C_{q}, C_{e}>0$ and $C_{q q}, C_{e e} \geq 0$. The sign of $C_{q e}$ depends on whether $e$ and $q$ are complements $\left(C_{q e}<0\right)$ or substitutes $\left(C_{q e}>0\right)$ in the physician disutility function. The participation constraint is then $U \geq 0$, which in equilibrium binds with equality.

The incentive compatibility constraint requires that the policy maker incorporates the physician best response to any given contract $(R, r, p)$ into the decision-making process. The physician best response can be described by the first-order conditions to the problem of choosing $(q, e)$ to maximize $U$ given the compensation contract. For the interior solution, these conditions are:

$$
\begin{aligned}
& \alpha B_{q}(q *, e *)+r-C_{q}(q *, e *)=0 \\
& \alpha B_{e}(q *, e *)+p-C_{e}(q *, e *)=0
\end{aligned}
$$

The solution to these two conditions is the physician best response functions $q(r, p)$ and $e(r, p) .{ }^{11}$ It is straightforward to show, using Cramer's rule, that $\partial q / \partial r=\left(C_{e e}-\alpha B_{e e}\right) / D>0$ and $\partial e / \partial p=\left(C_{q q}-\alpha B_{q q}\right) / D>0$, where $D=U_{q q} U_{e e}-U_{e q}{ }^{2}>0$ by the second-order necessary condition. Therefore, as expected, the physician provision of quantity and quality depends positively on their own prices. In addition, it is easy to show that $\partial e / \partial r=\partial q / \partial p=\left(\alpha B_{e q}-C_{e q}\right) / D$. The sign of this parameter is, in general, ambiguous and depends on whether $q$ and $e$ are complements or substitutes in the patient benefit and physician disutility functions. To gain some intuition, consider the standard case of effort substitution $\left(C_{e q}>0\right)$ where $q$ and $e$ compete for physician time. In this case, $\partial e / \partial r<0$ as the physician re-allocates his time from quality to quantity as the marginal return to quantity increases. This opportunity cost explanation is the only mechanism through which $r$ affects $e$ when the physician does not care about the patient's benefit $(\alpha=0)$. When the physician is altruistic, the negative effect of $r$ on $e$ due to the opportunity cost is amplified by the physician's concern for the patient if $q$ and $r$ are also substitutes in the production of health $\left(B_{e q}<0\right)$. In the opposite case, the physician's concern for the patient mitigates the negative effect of $r$ on $e$ and the net effect depends on the relative magnitudes of $\alpha, B_{e q}$ and $C_{e q}$.

Using the physician participation and incentive compatibility constraints, the policy maker's objective function can be expressed as:

$$
W=(1+\alpha) B(q(r, p), e(r, p))-C(q(r, p), e(r, p))
$$

The first-order condition for the quality bonus $p$ is then equal to:

\footnotetext{
${ }^{11}$ Note that $q$ and $e$ do not depend on the fixed payment, $R$, which plays a role only in the participation constraint.
} 


$$
\left[(1+\alpha) B_{q}-C_{q}\right] \partial q / \partial p+\left[(1+\alpha) B_{e}-C_{e}\right] \partial e / \partial p=0
$$

Using the first-order conditions in Equations (4) and (5) for the physician's problem and the fact that $\partial e / \partial r=\partial q / \partial p$, Equation $(7)$ can be expressed as:

$$
p=B_{e}+\left(B_{q}-r\right)(\partial e / \partial r) /(\partial e / \partial p)
$$

This equation relates the optimal size of P4P incentive $p$ to the degree of supply-side cost-sharing $r$. Given that $\partial e / \partial p>0$, this relation depends critically on the sign of $\partial e / \partial r$, which is a priori ambiguous, as we discussed earlier. In our empirical analysis, we aim to determine the sign of $\partial e / \partial r$ using the variation in physician response $(e)$ to the DMI between physicians practicing in an enhanced fee-for-service model $(r=1)$ and a blended capitation model $(0<r<1)$. We describe these two payment models and the DMI in more detail in the next section.

\section{INSTITUTIONAL BACKGROUND}

Until the early 2000s, almost all primary care physicians in Ontario practiced in a traditional fee-for-service model. In response to long-standing criticisms of this model, the government sequentially introduced a variety of new payment models. ${ }^{12}$ The common elements in these models include patient enrolment, extended hours and eligibility for a set of performance-based incentives, such as preventive care bonuses, special payments for providing targeted services, incentives to enrol patients with no regular family doctor and chronic disease management incentives. The main difference between the new models is in their base compensation, with two main options of fee-for-service and capitation.

Currently, approximately $80 \%$ of primary care physicians participate in the various new payment models. In this study, we focus on the two most prevalent new models, known as the FHG and the FHO. As of March 2011, there were more than 6500 physicians practicing in these two models, comprising approximately $60 \%$ of all primary care physicians in Ontario. The FHG is an enhanced fee-for-service model that was introduced in 2003. In this model, physicians receive a full fee-for-service value for services provided to their enrolled patients $(r=1)$, in addition to a premium for selected comprehensive care services. The FHO is a blended capitation model that was introduced in 2007. In this model, physicians receive an age- and sex-adjusted capitation rate for each enrolled patient $(R)$ and a discounted fee-for-service value for selected services $(r=0.15){ }^{13}$

The FHG and FHO models are identical in almost all other aspects, including the eligibility for the DMI. The DMI was introduced on April 1, 2006 in response to several concerns related to the management of diabetic patients. Specifically, prior to 2006, primary care physicians were compensated through a variety of fee codes for services provided to diabetic patients, such as the intermediate assessment and the diabetic management assessment. These codes paid physicians for services provided during the patient visit, but not for services provided for an extended period. As a result, this fee-for-service payment method did not explicitly encourage a planned approach to the ongoing management of diabetic patients. In addition, none of the existing codes required that the physician complies with all of the best clinical practice guidelines, such as those recommended by the Canadian Diabetes Association. ${ }^{14}$

In contrast, the DMI is paid for services provided to diabetic patients for the previous 12 months. Specifically, the DMI is paid for a planned, ongoing management of diabetic patients according to elements required by the Canadian Diabetes Association Clinical Practice Guidelines. These elements include '(a)

\footnotetext{
${ }^{12}$ For an overview of these new models, see for example Glazier et al. (2009), Kantarevic et al. (2011), and Li et al. (2011).

${ }^{13}$ A more detailed description of the payment mechanism in these two models is presented in Kantarevic and Kralj (2013) and in Appendix C.

${ }^{14}$ The diabetic management assessment requires that a physician complies with a subset of the guidelines specified by the CDA. This subset includes elements described in part (a) for the DMI, discussed later in this section.
} 
tracking lipids, cholesterol, $\mathrm{HbA1C}$, blood pressure, weight and body mass index and medication dosage; (b) discussion and offer of preventive measures including vascular protection, influenza and pneumococcal vaccination; (c) health promotion counselling and patient self-management support; (d) tracking of albumin to creatinine ratio, (e) discussion and offer of referral for dilated eye examination; and (f) foot examination and neurologic examination. ${ }^{15}$ To meet these guidelines, the physician must see the patient at least twice during the last 12 months. Physicians who meet the requirements may claim a code Q040 and receive an annual bonus of $\mathrm{C} \$ 60$ per patient. ${ }^{16}$ This bonus is payable in addition to the existing codes for services provided during the patient visit.

When it was introduced, the DMI was restricted to services provided by physicians in the patient enrolment models to their enrolled patients. As of April 1, 2009, this restriction was removed and eligibility was extended to all family physicians and both enrolled and non-enrolled patients. At the same time, the value of the DMI increased from $\mathrm{C} \$ 60$ to $\mathrm{C} \$ 75$ per patient.

As mentioned previously, our main empirical goal is to determine how the reimbursement rate affects the physician quality effort $(\partial e / \partial r)$. To do so, we use the variation in $r$ between the FHG and the FHO models to identify its effect on the physician response to the DMI $(e)$. Again, this comparison is particularly appealing because other payment elements, including the DMI, are nearly identical between the two models. ${ }^{17}$ However, a simple comparison between the two models may not be appropriate because physicians freely choose which model to join. This voluntary participation raises concerns about the selection bias if, as expected, factors that affect physician participation in a model also affect their response to the DMI. In the next section, we present our empirical approach to dealing with this potential problem.

\section{DIFFERENCE-IN-DIFFERENCE MATCHING}

\subsection{Parameter of Interest}

We wish to evaluate the difference in the physician response to the DMI between physicians participating in the FHG and FHO models. This evaluation problem can be studied within a potential outcomes framework ${ }^{18}$ in which we can precisely define the parameter of interest and clarify the assumptions needed to identify it.

Consider a simple setup in this framework with two periods and treatment in the second period only. Specifically, let $t=0$ denote the period before the introduction of the FHO model and let $t=1$ denote the period after its introduction. In addition, let $d_{i t}$ denote the treatment indicator for whether physician $i$ participates in the FHO model at time $t$. In this setup, $d_{i 0}=0$ for all physicians, $d_{i 1}=0$ for the FHG physicians and $d_{i 1}=1$ for the FHO physicians. Lastly, let $y_{i t}^{1}$ and $y_{i t}^{0}$ denote the potential outcomes (i.e. the physician response to the DMI) conditional on participating in the FHO and FHG models, respectively. For each physician, we can observe only $y^{1}$ or $y^{0}$ at any time. This observed outcome can be expressed as $y_{i t}=d_{i t} y^{1}{ }_{i t}+\left(1-d_{i t}\right) y_{i t}^{0}$.

Given this setup, we can precisely define any parameter we wish to study. In the literature, two commonly studied parameters are the mean effect of treatment (ATE) and the mean effect of treatment on the treated (ATT). ${ }^{19}$ In this article, we focus on the ATT because its identification requires much weaker assumptions than the identification of the ATE, as we discuss in the following paragraphs. In addition, given the voluntary participation in the new models, the ATE may be less policy relevant. In our setup, the ATT can be defined as $E\left[y_{i 1}^{1}-y_{i 1}^{0} \mid d_{i 1}=1\right]$, which represents the mean difference between actual and potential outcomes for the

\footnotetext{
${ }^{15}$ Schedule of Benefits, Physician Services under the Health Insurance Act (September 1, 2011), Ontario Ministry of Health and Long-term Care, page A39.

${ }^{16} \mathrm{As}$ a reference, this is equivalent to the fee for about two regular office visits (i.e. intermediate assessments).

${ }^{17}$ The minor differences include the Group Management and Leadership funding and the eligibility for the Continuing Medical Education grants, which apply only to the FHO model. However, these elements for nonclinical work represent a minor source of income for physicians participating in the FHO model.

${ }^{18}$ This model is also known as the Rubin causal model. See, for example, Rosenbaum and Rubin (1983, 1985).

${ }^{19}$ See, for example, Blundell and Costa Dias (2009) and Imbens and Wooldridge (2009).
} 
group of treatment physicians. One limitation of this definition is that it uses data from the posttreatment period only. To exploit data from both pretreatment and posttreatment periods, we use an equivalent definition of the ATT that can be expressed as:

$$
\mathrm{ATT} \equiv E\left[y^{1}{ }_{i 1}-y^{0}{ }_{i 0} \mid d_{i 1}=1\right]-E\left[y^{0}{ }_{i 1}-y^{0}{ }_{i 0} \mid d_{i 1}=1\right]=E\left[\Delta y_{i t} \mid d_{i 1}=1\right]-E\left[\Delta y^{0}{ }_{i t} \mid d_{i 1}=1\right] .
$$

\subsection{Identification Assumptions}

Without further assumptions, the ATT cannot be identified because we only observe $E\left[\Delta y_{i t} \mid d_{i 1}=1\right]$ but not the counterfactual outcome $E\left[\Delta y_{i t}^{0}{ }_{i t} d_{i 1}=1\right]$. In this study, we construct this missing counterfactual using the sample of comparison FHG physicians and estimate the ATT using the difference-in-difference (DD) matching estimators. $^{20}$

The identification of the ATT in the DD matching framework relies on two main assumptions. The first assumption, known as the conditional independence assumption (CIA), requires that

$$
E\left[\Delta y_{i t}^{0} \mid X_{i}, d_{i 1}=1\right]=E\left[\Delta y^{0}{ }_{i t} \mid X_{i}, d_{i 1}=0\right] .
$$

where $X_{i}$ is an appropriate set of observable covariates unaffected by treatment. This assumption states that, conditional on $X$, the mean change in potential outcomes for the treating physicians had they not joined the FHO model would be the same as the mean change in actual outcomes for the comparison FHG physicians. The CIA is a rather strong condition, but its plausibility in our study comes from the fact that it only needs to hold after unobserved time-invariant individual characteristics that affect both treatment and outcomes have been settled. Furthermore, because we focus on the ATT and not the ATE, the CIA needs to hold only for $\Delta y^{0}$ and not for $\Delta y^{1}$. Thus, the DD matching estimators that we implement allow for selection on fixed unobservable characteristics and on potential treatment outcomes. ${ }^{21}$

In practice, matching on all variables in $X$ becomes impractical as the number of covariates increases. Rosenbaum and Rubin (1983) show that if $\Delta y^{0}$ is the mean independent of treatment status given $X$, then it is also the mean independent of treatment status given $p\left(X_{i}\right)=\operatorname{Pr}\left(d_{i 1}=1 \mid X_{i}\right)$, where $p\left(X_{i}\right)$ is known as the propensity score. As a consequence, matching can be carried out using the propensity score alone instead of using all variables in $X$ and the CIA in Equation (10) can be replaced by

$$
E\left[\Delta y^{0}{ }_{i t} \mid p\left(X_{i}\right), d_{i 1}=1\right]=E\left[\Delta y^{0}{ }_{i t} \mid p\left(X_{i}\right), d_{i 1}=0\right] .
$$

The second assumption required for identifying the ATT in the DD matching models is that

$$
\operatorname{Pr}\left(d_{i 1}=1 \mid X_{i}\right)<1 .
$$

This assumption, known as the common support or overlap assumption, requires a positive probability of observing comparison physicians at each level of $X$. Note that we do not require that $\operatorname{Pr}\left(d_{i 1}=1 \mid X_{i}\right)>0$ because we focus on the ATT and not the ATE.

\subsection{Alternative DD Matching Estimators}

The alternative DD matching estimators that we consider in this study can be represented by the following general form:

\footnotetext{
${ }^{20}$ See, for example, Heckman, Ichimura, and Todd (1997, 1998), Smith and Todd (2005), and Ham et al. (2011). For implementation in STATA, see Leuven and Sianesi (2003) and Becker and Ichino (2002).

${ }^{21}$ That is, our identification strategy does not require that $E\left[\Delta y^{1}{ }_{i t} \mid X_{i}, d_{i 1}=1\right]=E\left[\Delta y^{1}{ }_{i t} \mid X_{i}, d_{i 1}=0\right]$ because we focus on the ATT and not the ATE.
} 


$$
\overline{\mathrm{ATT}}=n^{-1} \sum_{i}\left\{\Delta y_{i t}-\sum_{j} w(i, j) \Delta y_{j t}\right\}
$$

where $i$ and $j$ denote, respectively, the treatment and comparison physicians in the region of common support, $n$ is the number of treatment physicians in the region of common support, and $w(i, j)$ are the matching weights with $\Sigma_{\mathrm{j}} w(i, j)=1$. Therefore, the DD matching estimators construct the missing counterfactual outcome $\Delta y^{0}$ for each treatment physician $i$ by taking a weighted average of actual outcomes for comparison physicians who are matched to physician $i$.

Alternative matching estimators differ in how they construct the matching weights. We consider three commonly used matching estimators: nearest neighbour, conventional kernel and local linear kernel.

In the nearest neighbour estimator, each treatment physician is matched on the propensity score to the nearest comparison physician. The weighting scheme for this estimator assigns the weight of one to the closest comparison physician and the weight of zero to all other comparison physicians. In a sampling with a replacement version of this estimator, which we implement, a single comparison physician can be matched to more than one treatment physician. This is, in general, preferred to the sampling with no replacement if the distribution of propensity scores is very different between the treatment and the comparison groups. ${ }^{22}$

The nearest neighbour estimator is, in general, inefficient because it matches each treatment physician to a single comparison physician. This may be partially addressed by expanding the matched comparison group to $n>1$ physicians, in which case each matched comparison physician receives an equal weight of $1 / n$. However, this weighting scheme is problematic because close and distant matches receive the same weight in constructing the missing counterfactual. The conventional kernel estimator addresses this problem by matching all comparison physicians to each treatment physician and assigning a higher weight to comparison physicians closer to the matched treatment physician. Specifically, the weight that each comparison physician receives is equal to $w(i, j)=G\left(z_{j}\right) / \sum G\left(z_{j}\right)$, where $G($.$) is the kernel function, z_{\mathrm{j}}=\left(p_{i}-p_{j}\right) / h$ is the standardized distance in the propensity score between treatment physician $i$ and comparison physician $j$, and $h$ is the bandwidth. To implement the kernel estimator, the kernel function and the bandwidth must be specified. As our baseline case, we used the bi-weight kernel, which is equal to $15 / 16\left(z^{2}-1\right)^{2}$ for $|z|<1$ and 0 otherwise. As a specification check, we also explore several alternative kernels. For the bandwidth selection, we use Silverman's (1986) optimal plug-in selector, which produces the bandwidth of approximately 0.1 in our application, but we also experiment with alternative bandwidth values. ${ }^{23}$

The conventional kernel estimator constructs the missing counterfactual for each treatment physician nonparametrically as the weighted average of $\Delta y^{0}$ among the comparison physicians, which can be interpreted as a kernel-weighted regression of $\Delta y^{0}$ on a constant. The local linear kernel extends this regression model to include a linear term in $p_{i}-p_{j}$, which is helpful whenever comparison group observations are distributed asymmetrically around the treatment observations. ${ }^{24}$ Given the more desirable properties of this estimator compared with the conventional kernel and nearest neighbour, we use the local linear kernel as our baseline estimator.

\subsection{Standard Error Estimation}

Because of the complexity of the propensity score matching, most empirical studies rely on bootstrapping to compute the standard errors for the effect of treatment. This approach is expected to work well for the kernel and local linear kernel matching estimators but it is, in general, not valid for the nearest neighbour because of its extreme nonsmoothness (Abadie and Imbens, 2008). In implementing the bootstrap, we choose the

\footnotetext{
${ }^{22}$ See, for example, Dehejia and Wahba (2002).

${ }^{23}$ This bandwidth selector is described in detail in Appendix A.

${ }^{24}$ For example, Fan $(1992,1993)$ shows that the local linear estimator has a faster rate of convergence near boundary points and greater robustness to different data design densities than the conventional kernel estimator.
} 
optimal number of repetitions using the three-step methodology developed by Andrews and Buchinsky $(2000,2001) .^{25}$ In our application, this optimal number of repetitions is approximately 200.

\section{DATA}

The data come from several administrative sources maintained by the Ontario Ministry of Health and Longterm Care. Specifically, the Corporate Provider Database provides information on physician affiliation with a patient enrolment model, the Client Agency Program Enrolment database provides the list of all enrolled patients and the Ontario Health Insurance Plan database provides detailed, claim-level data on physician services provided to each patient. These sources can be linked together using encrypted physician and patient numbers to construct a comprehensive database that includes almost all family physicians and enrolled patients in Ontario and their entire profile of medical services.

The study period for our analysis includes fiscal years 2006 and 2010, one year before and three years after the FHO model was introduced in Ontario. ${ }^{26}$ For these two years, we focus on a cohort of physicians affiliated with the FHG model as of April 1, 2006. This cohort includes 4455 physicians, or approximately $40 \%$ of all primary care physicians in Ontario. Of this cohort, 441 physicians ceased to practice in Ontario between 2006 and 2010 for various reasons such as retirement and migration. Furthermore, 197 physicians switched to a patient enrolment model other than the FHO. These physicians were excluded from our analysis because our main focus is on the comparison between FHG and FHO physicians. Lastly, we excluded 162 physicians who had no enrolled patients in either 2006 or $2010 .{ }^{27}$ The final sample used for the analysis therefore includes 3655 physicians. ${ }^{28}$ Of this sample, approximately $42 \%$ of physicians switched to the FHO model by 2010 . For our purposes, these 1521 physicians are defined as treatment physicians, whereas the other 2134 physicians who remained in the FHG model are defined as comparison physicians.

The outcome of interest is measured in two complementary ways to capture the extensive and intensive margins of physician response to participating in the FHO model. On the extensive margin, we use a binary indicator for whether the physician participated at all in the DMI (i.e. whether the physician provided any Q040 services). One important advantage of using this measure is that it is expected to be measured with virtually no error. In addition, the results concerning this outcome may be particularly informative if factors that affect the decision to participate differ from factors that affect the decision on how many Q040 services to provide conditional on participation. On the intensive margin, we use the percentage of enrolled diabetic patients who received Q040 services. ${ }^{29}$ This measure is appealing because it reflects the targeted patient population. In addition, if the measure is interpreted as a probability that an enrolled diabetic patient receives

\footnotetext{
${ }^{25}$ This methodology is described in detail in Appendix B.

${ }^{26}$ We do not use data for the intervening years (2007-2009) for two main reasons. First, consistent with most empirical studies using the difference-in-difference matching, we need only one period before and one period after the policy change to implement this methodology. Second, the transition to the FHG model had matured by 2006 and the transition to the FHO model had matured by 2010 . The intervening years represent a period of rapid transition to the FHO model that may reflect a relatively short-term effect. Nevertheless, in Section 6.3, we provide some evidence on the dynamics of this effect in the intervening years by separately studying the cohorts of physicians who switched to the FHO model in each of 2008, 2009 and 2010.

${ }^{27}$ Unfortunately, it is difficult to determine the effect of these exclusions on our results. For physicians not present in 2010 , we cannot calculate changes in outcomes because we have only one observation per physician; for physicians who switched to other models, it is difficult to disentangle the effect of these models from the supply-side cost-sharing that we are interested in; and for the physicians with no enrolled patients, we cannot calculate one of our outcomes (the percentage of enrolled diabetic patients with the DMI), as we explain later in this section.

${ }^{28}$ Our actual estimation sample is slightly smaller (3588 physicians) because 67 comparison physicians could not be matched to any treatment physicians due to their low propensity scores.

${ }^{29}$ To identify diabetic patients, we use a methodology similar to that used by the Institute for Clinical and Evaluative Studies (2003). Specifically, the patients are identified as diabetic patients if they had any services over the last year with the Diabetes Mellitus ICD-10 diagnosis code or using fee codes that are provided exclusively to the diagnosed diabetic patients (the full list is available upon request). Using this methodology, we identified 724,237 diabetic patients in 2006 and 850,067 diabetic patients in fiscal year 2010 , which is within the range of published estimates.
} 
the DMI, it is invariant to how many total patients are enrolled with the physician and/or what percentage of enrolled patients has diabetes.

The set of covariates includes matching variables that we expect to belong to the propensity score model. The choice of the appropriate matching variables is critical for consistently estimating the treatment effect, ${ }^{30}$ which makes matching particularly attractive in our study because we have access to rich data on physician practices in the pretreatment period. Specifically, the included matching variables are related to (1) physician characteristics (physician age, sex and experience with the patient enrolment models (as measured by the number of days in the FHG model as of April 1, 2006)); (2) practice characteristics (the geographic location of practice, the number of enrolled patients, the annual number of patient visits and the number of other physicians in practice); (3) patient characteristics (the patient complexity (as measured by the risk-adjustment factors based on patients' age and gender) and the share of enrolled diabetic patients); (4) the expected income gain (estimated using the actual service and patient profiles in the fiscal year 2006 and the administrative payment rules in the FHG and FHO models ${ }^{31}$ ); and (5) past outcomes (an indicator for physician participation in the DMI in the fiscal year 2006, the percentage of enrolled diabetic patients who received the DMI in the fiscal year 2006). To ensure that the included covariates are not determined by treatment, all of the variables are measured before the introduction of the FHO model.

Descriptive statistics for the sample included in the analysis are presented in Table I. The first two columns contain variable names and definitions. The next three columns present the means for the entire sample, the treatment sample and the comparison sample, respectively. The last column presents the difference in means between treatment and comparison physicians. Standard errors for the sample means are presented in parentheses.

The top panel of Table I shows the outcomes of interest. On the intensive margin, the percentage of enrolled diabetic patients who received Q040 services was 22\% in the fiscal year 2006 and 34\% in the fiscal year 2010. This outcome was significantly larger for the treatment physicians in both years, with the difference growing over time from approximately $8 \%$ in 2006 to approximately 13\% in 2010. The simple difference-in-difference estimate of the FHO effect is approximately 5\% and it is statistically significant. On the extensive margin, approximately $49 \%$ of sample physicians provided Q040 services in 2006 and 68\% in the fiscal year 2010. Again, this outcome is significantly larger for the treatment physicians in both years, with the difference growing from approximately $13 \%$ in 2006 to approximately $21 \%$ in 2010 . Furthermore, the simple difference-in-difference estimate of approximately $8 \%$ is statistically significant. These unadjusted comparisons of outcome suggest that the treatment physicians responded to the DMI more than the comparison physicians on both extensive and intensive margins.

The bottom panel of Table I shows the distribution of covariates across the two groups of physicians as of the fiscal year 2006. These statistics indicate that the treatment physicians are, on average, two years younger and approximately $2 \%$ less likely to live in the Toronto region. In addition, the treatment physicians enrol more patients, practice in smaller groups, provide fewer annual visits and have been affiliated with the FHG model for a longer time. Perhaps most significantly, the expected income gain from joining the FHO model is approximately $\mathrm{C} \$ 57,000$ for the treatment physicians and approximately less than $\$ 15,000$ for the comparison physicians. All of these differences are statistically significant and suggest that physicians who joined the FHO model were a selected, nonrandom group of FHG physicians. This selection on observed covariates may also be indicative of selection on unobserved characteristics. These preliminary results confirm the need to address the potential selection bias when estimating the effect of participating in the FHO model.

\section{RESULTS}

We present our results in two steps. In the first step, we present the propensity scores that are estimated using the logistic model on the sample of FHG physicians in 2006. In this model, the dependent variable is an

\footnotetext{
${ }^{30}$ See, for example, Heckman, Ichimura, and Todd $(1997,1998)$ and Smith and Todd (2005).

${ }^{31}$ See Appendix C for details. 


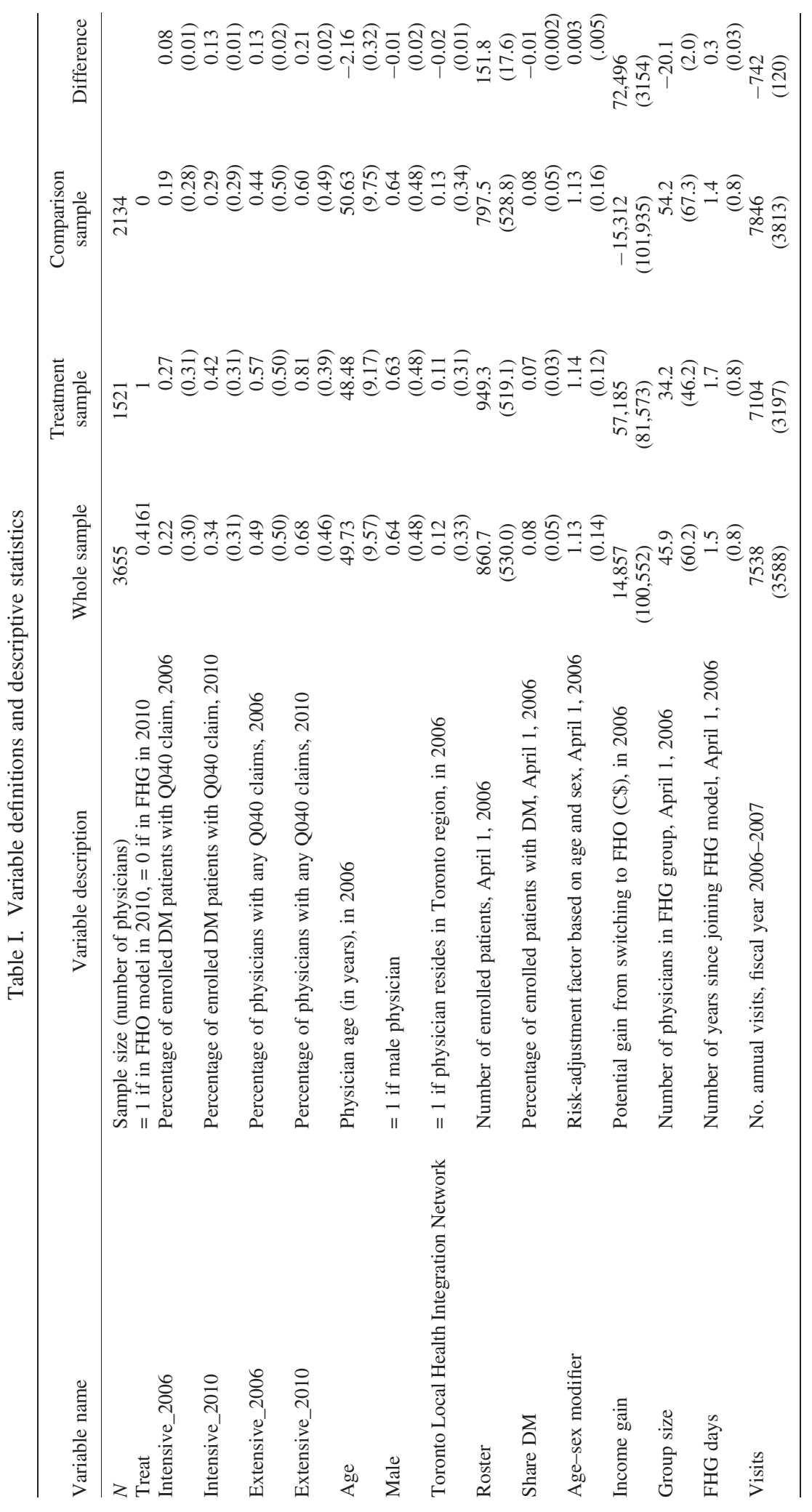


indicator equal to 1 if the physician ever joined the FHO model between 2006 and 2010, and 0 otherwise, and the set of covariates include those related to physician, practice and patient characteristics, the expected income gain, and the past outcomes, all measured as of 2006, as described in Section 5. In the second step, we present the DD matching estimates in which the outcome variables are (1) the change in the percentage of diabetic patients who received the DMI and (2) the change in the physician participation status in the DMI incentive, as described in Section 4.

\subsection{Propensity Scores}

Table II presents the propensity score logit estimates for participation in the FHO model. With the exception of gender, group size and the intensive measure of past outcomes, all coefficients are statistically significant. ${ }^{32}$ However, some coefficients do not have signs expected from the descriptive statistics reported in Table I. This is not surprising because some covariates are highly correlated, such as the number of enrolled patients and the number of annual visits. In addition, this is not a serious concern because the propensity score model does not necessarily represent a structural behavioural relationship because its main role in matching is to provide a good model for predicting treatment.

The estimated model has a good fit. The likelihood ratio test clearly rejects the hypothesis that included variables are jointly insignificant. ${ }^{33}$ In addition, McFadden's $R^{2}$ is approximately $0.24 .{ }^{34}$ Furthermore, the model correctly predicts treatment for approximately $72 \%$ of the sample physicians. This prediction metric is constructed by comparing the actual treatment status of each physician to their estimated probability of treatment. A prediction is considered to be correct if the estimated propensity score is higher than 0.42 for the treatment physician and lower than 0.42 for the comparison physician, in which 0.42 represents the percentage of sample physicians in the treatment group.

We chose the functional form of the variables included in the model to ensure that they are distributed similarly across the treatment and matched comparison physicians using balancing tests originally proposed by Rosenbaum and Rubin (1985). Specifically, for a given functional form, we tested whether our empirical model balanced the sample via paired $t$ tests and joint $F$ tests. The paired $t$ tests examine whether the mean of each covariate for the treatment group is equal to that of the matched comparison sample. The joint $F$ tests examine whether, at each quintile of the propensity score distribution, the mean of all covariates are jointly different between treatment and comparison physicians.

Table III shows these balancing tests, using the full sample of treatment physicians and matched samples of comparison physicians obtained using the nearest neighbour matching. The top panel shows the paired $t$ tests. These tests indicate that matching balances the two groups of physicians on each pretreatment covariate quite well because none of the reported differences are significant at the standard test levels. The bottom panel shows the joint $F$ tests. For the middle three quintiles, the $F$ tests cannot reject the hypothesis that these covariates are jointly insignificant. However, the $F$ tests are significant at the first and fifth quintiles, unless further restrictions are imposed on the propensity score distribution. Specifically, the $F$ tests are insignificant at the standard test levels only when the sample excludes observations with propensity scores of lower than 0.05 and higher than 0.95. Rather than imposing this restriction on our analysis, we present all of our results using the unrestricted sample and conduct the analysis with the restricted sample as a specification check. ${ }^{35}$

Lastly, the estimated propensity scores can be used to evaluate the validity of the overlap assumption in our sample. Figure 1 presents the distribution of the propensity scores for the treatment and comparison physicians. This figure shows that the empirical support of the two distributions is very similar, although, as expected, the

\footnotetext{
${ }^{32}$ The quadratic forms for age, visits and roster size are all statistically significant.

${ }^{33}$ The LR $\chi^{2}$ statistic with $30 d f$ is approximately 1194 , with the associated $P<0.000$.

${ }^{34}$ This $R^{2}$ is calculated as $1-L(B) / L(0)$, where $L(B)$ denotes the fitted log-likelihood value of the model and $L(0)$ denotes the value of loglikelihood in a constant-only model. The lower and upper bounds of this pseudo $R^{2}$ are 0 and 1 , but this pseudo $R^{2}$ is not a measure of proportion of variance of the dependent variable explained by the model.

${ }^{35}$ Our main empirical results are not sensitive to this restriction. Results are available upon request.
} 
PAY FOR PERFORMANCE INCENTIVES AND PHYSICIAN PAYMENT MECHANISMS

Table II. Propensity score logit estimates for participation in FHO

\begin{tabular}{lcc}
\hline Variable & Coefficient & Standard Error \\
\hline Age & 0.0013 & 0.0386 \\
Age $^{2}$ & -0.0005 & 0.0004 \\
Age $\times$ male & 0.0151 & 0.0106 \\
Male & -0.1722 & 0.5173 \\
Roster & -0.2777 & 0.2667 \\
Roster $^{2}$ & $-0.0003^{* * * *}$ & 0.0001 \\
Visits & $0.1241^{* *}$ & 0.0548 \\
Visits & -0.1660 & 0.2650 \\
Group size & -0.0010 & 0.0008 \\
Income gain & $0.0126^{* * *}$ & 0.0010 \\
Income gain & $0.0008^{* * *}$ & 0.0004 \\
Age-sex modifier & $8.7542^{* * *}$ & 2.7239 \\
Age-sex modifier ${ }^{2}$ & $-3.0855^{* * *}$ & 1.0858 \\
FHG days & $0.0008^{* * *}$ & 0.0002 \\
Share DM & $-6.5936^{* * *}$ & 1.1581 \\
Intensive_2006 & 0.2085 & 0.2028 \\
Extensive_2006 & $0.2624 * *$ & 0.1173 \\
Constant & $-5.5839^{* * *}$ & 1.8782 \\
\hline
\end{tabular}

To improve readability, the coefficients on Roster,

Roster $^{2}$, Visits and Income gain have been multiplied by $10^{3}$ and the coefficients on Visits ${ }^{2}$ and Income gain ${ }^{2}$ by $10^{8}$.

The model also includes 14 indicators for Local Health Integration Networks.

The sample size is 3588 physicians. The likelihood ratio $\chi^{2}$ statistic is 1194 with $30 d f$. McFadden's pseudo $R^{2}$ is 0.24 .

$* * *$ Significance at the $1 \%$ level; **significance at the $5 \%$ level; and *significance at the $10 \%$ level.

Table III. Balancing tests

Paired $t$ tests

\begin{tabular}{|c|c|c|c|}
\hline & Difference: unmatched & Difference: matched & $P$ value of paired $t$ statistics \\
\hline Age & -2.16 & -0.07 & 0.85 \\
\hline Male & -0.01 & 0.02 & 0.17 \\
\hline Toronto & -0.02 & 0.01 & 0.25 \\
\hline Roster & 152 & -41 & 0.08 \\
\hline Visits & -742 & -215 & 0.07 \\
\hline Share DM & -0.01 & -0.001 & 0.46 \\
\hline Age-sex modifier & 0.00 & -0.001 & 0.74 \\
\hline Income gain & 72,496 & -958 & 0.74 \\
\hline Group size & -20.1 & 0.6 & 0.72 \\
\hline FHG days & 109 & 3.0 & 0.78 \\
\hline Intensive_2006 & 0.08 & -0.01 & 0.61 \\
\hline Extensive_2006 & 0.13 & -0.01 & 0.43 \\
\hline \multicolumn{3}{|l|}{$F$ test statistics } & $P$ value \\
\hline First quintile & 536 & 1.71 & 0.06 \\
\hline Second quintile & 596 & 0.46 & 0.94 \\
\hline Third quintile & 594 & 0.33 & 0.98 \\
\hline Fourth quintile & 595 & 1.30 & 0.21 \\
\hline Fifth quintile & 522 & 1.55 & 0.10 \\
\hline
\end{tabular}

All tests are based on nearest neighbour matching. The unmatched difference is the difference between the full sample of treatment and comparison physicians for each covariate, whereas the matched differences are for the full sample of treatment physicians and only the matched sample of comparison physicians.

treatment physicians have a higher average probability of joining the FHO model than the comparison physicians. However, the overlap assumption fails for a small number of physicians at the extremes of the propensity score distribution. Specifically, 36 comparison physicians had propensity scores that were lower 


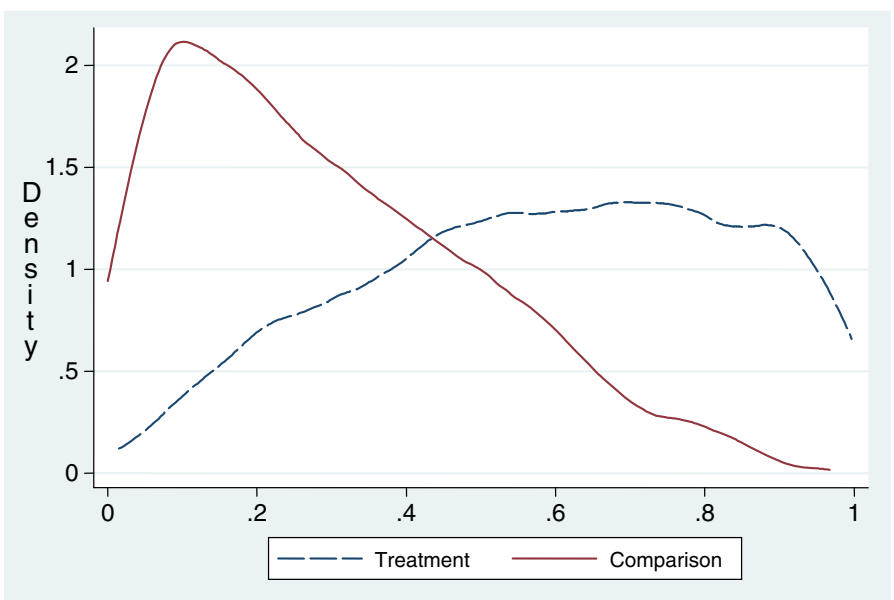

Figure 1. Distribution of estimated propensity scores

than the minimum score for treatment physicians $(0.015)$ and 52 treatment physicians had propensity scores that were higher than the maximum propensity score of comparison physicians (0.968). In our analysis, we impose the common support condition by excluding these 88 physicians, or approximately $1 \%$ of the sample from each tail of the propensity score distribution. ${ }^{36}$ In addition, as a specification check, we also exclude an additional $q$ percentage of treatment physicians for which the propensity score density of the comparison physicians is the lowest.

\subsection{Main Results}

Table IV presents our main results. The first row shows the baseline model, which is the local linear regression model using the bi-weight kernel, the bandwidth of 0.1 and the trimming level of $5 \%$. These results indicate that patients enrolled to the FHO physicians are approximately $8 \%$ more likely to receive the DMI services than patients enrolled to the FHG physicians. Similarly, physicians practicing in the FHO model are approximately $12 \%$ more likely to participate in the DMI than physicians practicing in the FHG model. Both of these effects are statistically significant. ${ }^{37}$ In addition, both effects are quite large compared with the pretreatment means of $22 \%$ and $49 \%$, respectively.

The remaining panels in Table IV show the sensitivity of our results to using alternative matching estimators, bandwidth values, kernel functions and trimming levels. With respect to the alternative estimators, we considered the nearest neighbour matching, with 1 and 10 neighbours, and conventional kernel estimator. In addition, we considered the bandwidth values that are half as large (0.05) and twice as large $(0.2)$ as our baseline value of 0.1. With respect to the kernel functions, the alternatives we considered were the Epanechnikov, normal, tri-cube and uniform functions. Lastly, we estimated the baseline model with no trimming and with the alternative trimming level of 0.1. Our baseline results are quite robust with respect to these alternative specifications. In each specification, the FHO's effect remains positive and statistically significant for both outcomes, and its magnitude is quite similar to our baseline estimates.

\footnotetext{
${ }^{36}$ Note that this is more than what is required by the overlap assumption in Equation (12), which only requires the exclusion of 52 physicians for which $p\left(d_{i t}=1 \mid X_{i}\right)=1$.

${ }^{37}$ Because the decision to join the FHO model is sometimes made at the group level, we also estimated the baseline model by bootstrapping the standard errors stratified at the group level. Our results are very similar to those presented here and are available upon request. However, note that it is not a priori clear whether clustering should be performed at the individual or group level, because the decision to join the FHO is sometimes made at the individual level, the patients are enrolled to the physician and not the group, and the DMI is paid to the physician and not the group.
} 
PAY FOR PERFORMANCE INCENTIVES AND PHYSICIAN PAYMENT MECHANISMS

Table IV. Difference-in-difference matching estimates of FHO's effect

\begin{tabular}{|c|c|c|}
\hline & Enrolled diabetic patients with DMI & Physicians with DMI \\
\hline Baseline model & $\begin{array}{l}0.0843 * * * \\
(0.0146)\end{array}$ & $\begin{array}{l}0.1153 \text { *** } \\
(0.0223)\end{array}$ \\
\hline \multicolumn{3}{|l|}{ Alternative estimators } \\
\hline Nearest neighbour (1 neighbour) & $\begin{array}{l}0.0972 * * * \\
(0.0182)\end{array}$ & $\begin{array}{l}0.1271^{* * * *} \\
(0.0284)\end{array}$ \\
\hline Nearest neighbour (10 neighbours) & $\begin{array}{l}0.0847 * * * \\
(0.0152)\end{array}$ & $\begin{array}{l}0.1108 * * * \\
(0.0236)\end{array}$ \\
\hline Kernel & $\begin{array}{l}0.0803 * * * \\
(0.0135)\end{array}$ & $\begin{array}{l}0.1147^{* * * *} \\
(0.0216)\end{array}$ \\
\hline \multicolumn{3}{|l|}{ Alternative bandwidth values } \\
\hline 0.05 & $\begin{array}{l}0.0846 * * * \\
(0.0142)\end{array}$ & $\begin{array}{l}0.1142 * * * \\
(0.0255)\end{array}$ \\
\hline 0.20 & $\begin{array}{l}0.0836 \text { *** } \\
(0.0148)\end{array}$ & $\begin{array}{l}0.1086^{* * * *} \\
(0.0232)\end{array}$ \\
\hline \multicolumn{3}{|l|}{ Alternative kernel functions } \\
\hline Normal & $\begin{array}{l}0.0815 * * * \\
(0.0145)\end{array}$ & $\begin{array}{l}0.1076 * * * \\
(0.0231)\end{array}$ \\
\hline Uniform & $\begin{array}{l}0.0839 * * * \\
(0.0149)\end{array}$ & $\begin{array}{l}0.1127 * * * \\
(0.0227)\end{array}$ \\
\hline Epanechnikov & $\begin{array}{l}0.0841 * * * \\
(0.0147)\end{array}$ & $\begin{array}{l}0.1145^{* * *} \\
(0.0224)\end{array}$ \\
\hline Tricube & $\begin{array}{l}0.0830 \text { *** } \\
(0.0148)\end{array}$ & $\begin{array}{l}0.1095 * * * \\
(0.0234)\end{array}$ \\
\hline \multicolumn{3}{|l|}{ Alternative trimming levels } \\
\hline No trimming & $\begin{array}{l}0.0818 * * * \\
(0.0135)\end{array}$ & $\begin{array}{l}0.1067 * * * \\
(0.0219)\end{array}$ \\
\hline $10 \%$ & $\begin{array}{l}0.0760 * * * \\
(0.0130)\end{array}$ & $\begin{array}{l}0.1157 * * * \\
(0.0214)\end{array}$ \\
\hline
\end{tabular}

The baseline model is the local linear regression model, using the bi-weight kernel, a bandwidth of 0.1 and imposing a common support by dropping treatment observations whose propensity scores are higher than the maximum or lower than the minimum propensity scores of the comparison physicians and by dropping $5 \%$ of the treatment observations at which the propensity score density of the comparison observations is the lowest.

The sample size is 3588 physicians.

Bootstrap standard errors in parentheses, using 200 bootstrap repetitions.

$* * *$ Significance at the $1 \%$ level; ${ }^{* *}$ significance at the $5 \%$ level; ${ }^{*}$ significance at the $10 \%$ level.

\subsection{Specification Checks}

The CIA can never be directly verified because the counterfactual outcomes in the nontreatment state cannot be observed for any treatment physician. However, we conduct three specification checks to shed some light on the validity of this assumption.

The pretreatment test, originally proposed by Heckman and Hotz (1989), relies on data on outcomes in the pretreatment period and knowledge of future treatment status of sample physicians. The test is based on the idea that a consistent estimator applied to the pretreatment data should make the outcomes of future treatment and comparison physicians similar. The results from this test are presented in the second panel of Table V. For convenience, the first panel reproduces our baseline results from Table IV. These results indicate that our baseline estimator, the local linear regression, aligns the treatment and comparison physicians quite well in the pretreatment period. Specifically, the estimated coefficients on both outcomes are small and statistically insignificant, as would be expected if the CIA holds.

The second test is based on the idea that the treatment effect of joining the FHO model, if it exists, should be observed across successive cohorts of future treatment physicians. Note that this effect need not be identical across the cohorts, either because 'early adopters' are different from 'late adopters' or because it takes time to set up better care management processes. However, as long as the time investment is not too significant, the FHO's effect should be observed to some extent in all cohorts of treatment physicians over our sample 
Table V. Pretreatment effect and effect by year of switch

\begin{tabular}{lccc}
\hline & Sample & Enrolled diabetic patients with DMI & Physicians with DMI \\
\hline Baseline model & 3588 & $0.0843^{* * *}$ & $0.1153^{* * *}$ \\
& & $(0.0146)$ & $(0.0223)$ \\
Pretreatment effect & 3595 & $-0.0005(0.0233)$ & $-0.0141(0.0299)$ \\
Effect by year of switch & & $0.1018^{* * *}$ & 0.0899 \\
2008 cohort & 2444 & $(0.0330)$ & $(0.0843)$ \\
& & $0.0691^{* * *}$ & $0.1095^{* * *}$ \\
2009 cohort & 2816 & $(0.0164)$ & $(0.0247)$ \\
2010 cohort & 2482 & $0.0762^{* * *}$ & $0.1176^{* *}$ \\
& & $(0.0173)$ & $(0.0280)$ \\
\hline
\end{tabular}

For the baseline model, see note in Table IV.

The pretreatment effect specification uses the outcomes in and the baseline matching model.

The cohort for each year represents treatment physicians who joined FHO in that year. The set of comparison physicians is the same for each of the cohort models. Each row represents a separate model for each cohort.

Bootstrap standard errors in parentheses, using 200 bootstrap repetitions.

***Significance at the $1 \%$ level; **significance at the $5 \%$ level; *significance at the $10 \%$ level.

period. The results from this test are presented in the third panel of Table V. In our sample, there were three main cohorts of physicians joining the FHO model in 2008 (370 physicians), in 2009 (745 physicians) and in 2010 (406 physicians). To facilitate the comparison of estimates across cohorts, we used the same group of comparison physicians in each model. The results show a positive and significant FHO effect on the percentage of diabetic patients receiving DMI services for all three cohorts of treatment physicians, although the effect seems somewhat stronger for the 2008 cohort. On the other hand, the estimated effect on the probability of physician participation in the DMI is positive for all three cohorts, with a similar magnitude across cohorts and with our baseline estimates, although the effect is estimated imprecisely for the 2008 cohort. Again, these cohort-specific results are largely consistent with the causal interpretation of FHO's effect.

Lastly, we examine the sensitivity of our results to the choice of matching variables included in the propensity score model. As mentioned earlier, this choice is critically important for consistently estimating the ATT. $^{38}$ At the same time, this choice is quite difficult because it must simultaneously satisfy the requirements of both the CIA and the common support assumption. In particular, the set of matching variables must be rich enough to ensure that the potential outcomes in the nontreated state $\left(y^{0}\right)$ are similar between the treatment and the comparison physicians, but including any additional variables will make the common support assumption more likely to fail. To examine this issue, we estimated our baseline model using the successively richer sets of matching variables. Specifically, we start with the model that includes only variables related to physician characteristics, and then successively add those related to practice characteristics, patient characteristics, the expected income gain and the past outcomes. The results of this analysis, presented in Table VI, indicate that the estimates are uniformly smaller whenever we use less than the full set of matching variables. ${ }^{39}$ At the same time, the estimates are positive and statistically significant in all models, suggesting that our results are not overly sensitive to these permutations of matching variables. Perhaps most significantly, our baseline results presented in Table IV depend most critically on the inclusion of two past outcomes. In fact, including only the past outcomes in the propensity score model produces estimates nearly identical to our

\footnotetext{
${ }^{38}$ For example, Heckman and Lozano (2004) show that bias may result if the conditioning set of variables is not the right and complete one. Specifically, if the relevant information is not all controlled for, adding additional relevant information, but not all that is required, may increase rather than reduce bias.

${ }^{39}$ Heckman et al. (1997) show that the bias of matching estimators need not vary monotonically with the number of matching variables included in the propensity score model.
} 
PAY FOR PERFORMANCE INCENTIVES AND PHYSICIAN PAYMENT MECHANISMS

Table VI. Choice of matching variables

\begin{tabular}{lcc}
\hline Set of matching variables & Enrolled diabetic patients with DMI & Physicians with DMI \\
\hline (1) Physician characteristics & $0.0585^{* * * *}$ & $0.0788^{* * * *}$ \\
(2) Practice characteristics + (1) & $(0.0114)$ & $(0.0170)$ \\
& $0.0567 * * *$ & $0.0650^{* * *}$ \\
(3) Patient characteristics + (2) & $(0.0134)$ & $(0.0224)$ \\
& $0.0488^{* * *}$ & $0.0429^{*}$ \\
(4) Expected income gain + (3) & $(0.0138)$ & $(0.0238)$ \\
& $0.0630^{* * *}$ & $0.0544^{*}$ \\
(5) Past outcomes + (4) & $(0.0245)$ & $(0.0332)$ \\
& $0.0843^{* * *}$ & $0.1153^{* * *}$ \\
\hline
\end{tabular}

For the baseline model, see note in Table IV.

Physician characteristics include age, sex and days in FHG model as of April 1, 2006; practice characteristics include geographic location, number of enrolled patients, number of annual visits and group size; patient characteristics include the average age-sex modifier and the percentage of enrolled patients that are diabetic; the expected gain is the calculated income gain from switching from the FHG to FHO model; and past outcomes include the percentage of enrolled diabetic patients that received DMI in 2006 and the percentage of physicians participating in the DMI in 2006. Further details are available in Section 5.Bootstrap standard errors in parentheses, using 200 bootstrap repetitions.

$* * *$ Significance at the $1 \%$ level; **significance at the $5 \%$ level; *significance at the $10 \%$ level.

baseline results. This finding is particularly comforting because the past outcomes could contain all the relevant information on the unobservable physician characteristics as they are partly determined by such factors. ${ }^{40}$

\subsection{Subgroup Analysis}

Our main results reported in Table IV represent the average effect of joining the FHO model. In this section, we examine how this effect varies for specific groups of physicians. The groups were defined using the pretreatment (2006) values for the following variables: share of enrolled patients with diabetes, expected income gain, share of enrolled diabetic patients with the DMI and percentage of physicians participating in the DMI ${ }^{41}$ For each variable, we split the sample into two groups using the median value of that variable in 2006 and then estimated the physician response to the DMI using the same baseline DD matching model as in Table IV.

The results, presented in Table VII, suggest that there is some heterogeneity in the physician response to the DMI. Specifically, FHO's effect is concentrated among physicians with no or weak participation in the DMI before the treatment period (i.e. those with low values of the pretreatment outcomes). ${ }^{42}$ In addition, the effect on the extensive margin (the physician participation in the DMI) is concentrated among physicians with a smaller share of enrolled patients with diabetes and those with a smaller expected income gain. On the other hand, the effect on the intensive margin (the share of diabetic patients receiving the DMI) is positive and statistically significant for groups with different pretreatment values of the expected income gain and the share of enrolled patients with diabetes.

\subsection{Effect on Quantity}

Although our main focus is on quality, our theoretical model also predicts that the quantity of medical care should be negatively related to the degree of supply-side cost-sharing $(\partial q / \partial r>0)$. This implies that physicians practicing in the blended capitation FHO model can be expected to provide fewer services than if they were

\footnotetext{
${ }^{40}$ In addition to these specification checks, we also estimated the standard difference-in-difference regression models, as well as the crosssection matching model. The results are qualitatively similar to those reported here.

${ }^{41}$ We have also analyzed the subgroups defined by physician age, gender, location of practice (urban versus rural) and experience with the new primary care models. The results, available upon request, indicate that there is some heterogeneity in the physician response to the DMI, but the estimated effect is positive and statistically significant for each physician group and for each outcome.

${ }^{42}$ This result is consistent with findings in the literature, e.g. Rosenthal et al. (2005) and Lindenauer et al. (2007).
} 
Table VII. Estimates of FHO's effect by subgroups

\begin{tabular}{|c|c|c|c|}
\hline & Sample & Enrolled diabetic patients with DMI & Physicians with DMI \\
\hline Baseline model & 3588 & $\begin{array}{l}0.0843 * * * \\
(0.0146)\end{array}$ & $\begin{array}{l}0.1153 * * * \\
(0.0223)\end{array}$ \\
\hline \multicolumn{4}{|c|}{ Share enrolled diabetic patients in 2006} \\
\hline Below median $(<8.89 \%)$ & 1793 & $\begin{array}{l}0.0913 * * * \\
(0.0168)\end{array}$ & $\begin{array}{l}0.1506 * * * \\
(0.0278)\end{array}$ \\
\hline Above median $(\geq 8.89 \%)$ & 1793 & $\begin{array}{c}0.0708^{*} \\
(0.0421)\end{array}$ & $\begin{array}{c}0.0260 \\
(0.0602)\end{array}$ \\
\hline \multicolumn{4}{|l|}{ Expected income gain in 2006} \\
\hline Below median $(<\mathrm{C} \$ 29,854)$ & 1794 & $\begin{array}{l}0.0926 \text { *** } \\
(0.0182)\end{array}$ & $\begin{array}{l}0.1573 * * * \\
(0.0316)\end{array}$ \\
\hline Above median $(\geq \mathrm{C} \$ 29,854)$ & 1794 & $\begin{array}{l}0.0811 \text { *** } \\
(0.0241)\end{array}$ & $\begin{array}{l}0.0847 * * \\
(0.0431)\end{array}$ \\
\hline \multicolumn{4}{|c|}{ Enrolled diabetic patients with DMI in 2006} \\
\hline Below median $(<2.78 \%)$ & 1794 & $\begin{array}{l}0.1170 * * * \\
(0.0214)\end{array}$ & $\begin{array}{l}0.1893 * * * \\
(0.0623)\end{array}$ \\
\hline Above median $(\geq 2.78 \%)$ & 1791 & $\begin{array}{c}0.0476 \\
(0.0541)\end{array}$ & $\begin{array}{c}0.0114 \\
(0.0185)\end{array}$ \\
\hline \multicolumn{4}{|l|}{ Physicians with DMI in 2006} \\
\hline No claims for DMI & 1802 & $0.1171 * * *(0.0203)$ & $0.2115 * * *(0.0447)$ \\
\hline Claims for DMI & 1784 & $0.0523 *(0.0277)$ & $0.0057(0.01450)$ \\
\hline
\end{tabular}

For the baseline model, see note in Table IV.

Bootstrap standard errors in parentheses, using 200 bootstrap repetitions.

$* * *$ significance at the $1 \%$ level; **significance at the $5 \%$ level; *significance at the $10 \%$ level.

practicing in the enhanced fee-for-service FHG model. To test this prediction, we obtained three measures of the quantity of medical care: annual services, annual visits and annual days of work. Using the logs of these outcomes as our dependent variables, we then implemented the same DD matching estimator as for our previous results for the DMI. The results, presented in Table VIII, confirm this prediction for the annual services and visits (a reduction of approximately 5\% and 9\%, respectively), but not for the annual days of work. The results indicate that the FHO physicians provide fewer services and visits per day, but work a similar number of days per year. These results are consistent with our behavioural model and lend further credence to the causal interpretation of our findings for the DMI.

\subsection{Policy Implications}

Our empirical results suggest that physicians in a blended capitation model are more responsive to the DMI than physicians in an enhanced fee-for-service model. In terms of our theoretical model, this result implies that

Table VIII. Estimates of FHO's effect on quantity

\begin{tabular}{lr}
\hline Quantity outcome & FHO effect \\
\hline Log of annual services & $-0.0512^{* *}$ \\
Log of annual visits & $(0.0206)$ \\
Log of annual days & $-0.0875^{* * *}$ \\
& $(0.0196)$ \\
\end{tabular}

The model used is the local linear regression model, with the bi-weight kernel, a bandwidth of 0.1 and imposing a common support by dropping treatment observation whose propensity score is higher than the maximum or lower than the minimum propensity score of the comparison physicians and by dropping $5 \%$ of the treatment observations at which the propensity score density of the comparison observations is the lowest.

The sample size is 3588 physicians.

Bootstrap standard errors in parentheses, using 200 bootstrap repetitions.

***Significance at the $1 \%$ level; **significance at the $5 \%$ level; *significance at the $10 \%$ level. 
the quality effort varies positively with the degree of supply-side cost-sharing $(\partial e / \partial r<0)$. Furthermore, given that $p=B_{e}+\left(B_{q}-r\right)(\partial e / \partial r) /(\partial e / \partial p)$ from Equation (8), this result implies that for a given compensation mechanism, the optimal size of $\mathrm{P} 4 \mathrm{P}$ incentive varies negatively with the degree of supply-side cost-sharing $(\partial p / \partial r>0)$.

The main policy implication of this result is that the design of $\mathrm{P} 4 \mathrm{P}$ programs must take into account the underlying physician payment mechanism. Our analysis shows that the P4P incentive should be higher when the degree of cost-sharing is lower. ${ }^{43}$ This argument must be interpreted with caution, however, because it strictly applies to comparing payment mechanisms with small differences in the cost-sharing parameter $r$. Therefore, it does not necessarily follow from this argument that the P4P incentives in the capitation model should be smaller than in the fee-for-service model. Rather, the argument is more policy relevant when contemplating changes in the size of $\mathrm{P} 4 \mathrm{P}$ incentives following small changes in the physician compensation mechanism. For example, the Ontario Ministry of Health and Long-term Care increased the cost-sharing parameter in the FHO model, known as the shadow billing rate, from 0.1 to 0.15 on October 1, 2010. Our analysis implies that this change should be accompanied with a corresponding increase in the DMI and other $\mathrm{P} 4 \mathrm{P}$ performance incentives.

A corollary of our results is that the quality bonus $p$ should be set above the marginal effect of quality on patient's health $\left(p>B_{e}\right)$ whenever there is an overprovision of medical services $\left(B_{q}<r\right)$. Similarly, $p<B_{e}$ whenever there is an underprovision of medical services $\left(B_{q}>r\right)$. The intuition for this result is simple. The introduction of a P4P program creates incentives to reallocate physician effort from 'quantity' to 'quality'. To the extent that there is an existing distortion in quantity, the quality bonus addresses the twin goals of improving quality and reducing distortions in quantity.

The possible distortions in the quantity of medical services may arise mainly because the policy maker takes the physician compensation mechanism as a given when introducing a new P4P program. Clearly, such distortion can be eliminated and welfare improved if the introduction of P4P programs is determined jointly with changes to the physician payment mechanisms. Such a wholesome approach to health care reform may be welfare improving because, as our analysis suggests, there exist important links between the physician response to the $\mathrm{P} 4 \mathrm{P}$ programs and the type of physician compensation mechanism.

\subsection{Limitations}

We conclude this section by discussing the three main limitations of our study. ${ }^{44}$ First, we documented that the capitation physicians are more likely to participate in the DMI than the fee-for-service physicians, and we interpreted this difference as a behavioural response to the difference in the supply-side cost-sharing. However, there are at least two alternative interpretations of these results. The first interpretation is that the results represent increased gaming behaviour by the FHO physicians because payment for the DMI is based on the self-reported physician claims. The second interpretation is that the results represent better information systems to monitor and manage diabetic patients acquired by the FHO physicians because of increased financial risk in the capitation model. Both of these interpretations are plausible and both require access to data unavailable to us to gauge their empirical importance. However, we suspect that neither of these interpretations has sufficient power to fully explain our results. For example, the extent of gaming and misreporting is importantly limited by the relatively small size of the DMI bonus and the relatively high expected cost of fraudulent behaviour. In addition, the fee-for-service system in Ontario, and in all other Canadian provinces, has been based on the self-reported physician claims since the introduction of Medicare and yet the reports of fraudulent behaviour are quite rare. Furthermore, the financial risk borne by the FHO physicians is limited to their participation in

\footnotetext{
${ }^{43}$ As one referee points out, this policy response can be viewed as rewarding bad behavior: physicians that stick with the fee-for-service model need a greater incentive to improve quality. An alternative policy response is that more physicians should be encouraged and/or coerced to join the FHO.

${ }^{44} \mathrm{We}$ thank two anonymous referees for raising these points.
} 
the FHO model, which can be terminated at any time and for any reason, without any cost, penalty or liability, by providing the government with 60 days' notice. Less radically, the FHO physicians can limit their financial risk by choosing to be compensated for their high-use patients through the fee-for-service system. Both of these arguments do not claim that gaming and better information system are not empirically relevant, but they significantly reduce the extent of their relevance on a priori grounds.

Second, the DMI rewards physicians for complying with the best practice guidelines for treating diabetic patients. Some critics may argue that quality should be measured instead in terms of improved patient outcomes. We lack data to assess the relationship between compliance with the DMI and patients' outcomes. However, we suspect that our results represent a genuine improvement in the quality of care for diabetic patients and not just the 'teaching to the test' effect given that the best practice guidelines were developed in large part based on their expected effect on patient outcomes. ${ }^{45}$ Nevertheless, we acknowledge that there is an ongoing debate about the relative merits of outcome-based and process-based measures of quality, and much remains to be learned.

Lastly, our empirical strategy and specification checks were developed to address the internal validity of our results. Therefore, our estimates of the average treatment effect on those treated might not be generalized to the entire population of Ontario primary care physicians, or to other jurisdictions with significantly different health care systems or to other P4P initiatives in Ontario. Further research is needed to determine the external validity of these results.

\section{CONCLUSION}

In this study, we compare physician response to the DMI, a new pay-for-performance incentive in Ontario, between physicians practicing in an enhanced fee-for-service model and in a blended capitation model. Using a comprehensive administrative data strategy and a difference-in-difference matching strategy, we find that physicians in a blended capitation model are more responsive to the DMI than physicians in an enhanced fee-for-service model. We show that for a given payment mechanism, this result implies that the optimal size of P4P incentives is negatively related to the degree of supply-side cost-sharing. These results suggest that the optimal design of $\mathrm{P} 4 \mathrm{P}$ programs is importantly linked to the physician payment mechanisms. More generally, our analysis suggests that a joint approach to both the physician payment reform and the design of P4P programs may be welfare improving.

Future research can build on our analysis in at least two ways. First, our analysis is based on two types of physician payment mechanisms (enhanced fee-for-service and blended capitation) and a single P4P incentive (the DMI). Future analysis can examine the physician response between these two models to other P4P incentives, such as preventive care bonuses and incentives to enrol new patients. Similarly, the difference in physician response to the $\mathrm{P} 4 \mathrm{P}$ incentives can be studied using other types of physician payment mechanisms, such as the traditional fee-for-service model and salary. Second, we studied the physician uptake of the DMI, which involves a planned, ongoing management of diabetic patients using the best practice clinical guidelines. Ultimately, the policy importance of this incentive is its effect on patients' health and health care costs. This remains a promising area for future research, following advances already made in the literature. ${ }^{46}$

\section{APPENDIX A: SELECTING THE BANDWIDTH VALUE}

Silverman's rule of thumb method consists of finding the bandwidth value, which minimizes the mean squared integrated error for the kernel density estimator and then replacing the unknown quantities with an estimate.

\footnotetext{
${ }^{45}$ For details, see Canadian Diabetes Association Clinical Practice Guidelines (2008).

${ }^{46} \mathrm{See}$, for example, Dusheiko et al. (2011). 
For example, taking the Gaussian kernel (which is identical to the standard normal pdf), the rule of thumb expression for the optimal bandwidth value is $1.06 \hat{\sigma} n^{-1 / 5}$, where $n$ is the sample size. The results of implementing this method in our study are given in Table A1.

Table A1. Silverman's rule of thumb bandwidth selector with Gaussian kernel

\begin{tabular}{lcr}
\hline & Enrolled diabetic patients with DMI & Physicians with DMI \\
\cline { 2 - 3 } Sample size & 3598 & 3655 \\
SD & 0.2829 & 0.4913 \\
Optimal bandwidth & 0.0583 & 0.1009 \\
\hline
\end{tabular}

\section{APPENDIX B: SELECTING THE NUMBER OF BOOTSTRAP REPETITIONS}

We closely follow Ham et al. (2011) in implementing the methodology developed by Andrews and Buchinsky (2000, 2001). Let $\theta$ be the ATT parameter identified by the matching estimator and let $\lambda$ be its standard error. Furthermore, let $B$ denote the number of repetitions and pdb the measure of accuracy, which is the percentage deviation of the bootstrap quantity of interest based on bootstrap repetitions from the ideal bootstrap quantity of interest for which $B=\infty$. The magnitude of $B$ depends on both the accuracy required and the data. If we required the actual percentage deviation to be less than $\mathrm{pdb}$ with a specified probability $1-\tau$, then the Andrews and Buchinsky method proposes a three-step method that takes pdb and $\tau$ as a given and provides a minimum number of repetitions $B^{*}$ to obtain the desired level of accuracy. We follow Ham et al. (2011) and set $(\mathrm{pdb}, \tau)=(10,0.05)$. In the first step, we calculate the initial number of repetitions $B_{1}=\operatorname{int}\left(10,000 * z_{1-\tau / 2}^{2} * 0.5 /\right.$ $\left.\mathrm{pdb}^{2}\right)$, where $z_{1-\tau / 2}$ is the $1-\tau / 2$ quantile of standard normal distribution. In our case, $B_{1}=193$. In the second step, we use the bootstrap results $\left\{\hat{\theta}: \hat{\theta}_{1}, \ldots, \hat{\theta}_{B 1}\right\}$ to calculate $\omega=\left(2+\gamma_{B}\right) / 4$, where $\gamma_{B}=\left(B_{1}-1\right)^{-1} \sum_{r}^{B 1}\left(\hat{\theta}_{r}-\mu_{B}\right)^{4} / s e_{B}^{4}-3$, where $\mu_{B}$ and $s e_{B}$ are the mean and standard deviation of $\{\hat{\theta}$ : $\left.\hat{\theta}_{1}, \ldots, \hat{\theta}_{B 1}\right\}$. The new number of repetitions is then calculated as $B_{2}=\operatorname{int}\left(10,000 * z_{1-\tau / 2}^{2} * \omega / \mathrm{pdb}^{2}\right)$. In the last step, the minimum number of repetitions is determined as $B^{*}=\max \left(B_{1}, B_{2}\right)$. The results of implementing this methodology in our study are given in Table B1.

Table B1. Andrews and Buchinsky $(2000,2001)$ Method for selecting bootstrap repetitions

\begin{tabular}{|c|c|c|c|c|}
\hline \multirow[b]{2}{*}{$\begin{array}{l}\text { Matching } \\
\text { model }\end{array}$} & \multicolumn{2}{|l|}{ Estimated $B_{2}$} & \multicolumn{2}{|c|}{ Optimal number $B^{*}$} \\
\hline & $\begin{array}{c}\text { Enrolled diabetic patients with } \\
\text { DMI }\end{array}$ & $\begin{array}{l}\text { Physicians with } \\
\text { DMI }\end{array}$ & $\begin{array}{c}\text { Enrolled diabetic patients with } \\
\text { DMI }\end{array}$ & $\begin{array}{l}\text { Physicians with } \\
\text { DMI }\end{array}$ \\
\hline LLR & 193 & 233 & 193 & 233 \\
\hline Kernel & 191 & 183 & 193 & 193 \\
\hline $\mathrm{NN}$ & 212 & 189 & 212 & 193 \\
\hline
\end{tabular}

LLR, local linear regression; NN, nearest neighbour.

\section{APPENDIX C: EXPECTED INCOME GAIN}

In our analysis, we estimated the expected difference in income for a cohort of FHG physicians in 2006 between what they actually earned in 2006 and what they would have hypothetically earned if they practiced in the FHO model. The actual base compensation for these physicians can be represented as $I_{\mathrm{FHG}}=1.1 p_{1} q_{1} m+$ $p_{1} q_{1} n+p_{2} q_{2}(m+n)$, where $q_{1}$ represents services eligible for the $10 \%$ comprehensive care premium, $q_{2}$ represents all other services, $m$ is the number of enrolled patients and $n$ is the number of non-enrolled patients. In contrast, the hypothetical income for these physicians if they had practiced in the FHO model can be represented as $I_{\mathrm{FHO}}=R m+0.1 p_{1} q_{1} m+p_{2} q_{2}(m+n)+\min \left\{p_{1} q_{1} n, z\right\}$, where $R$ is the age- and sex-adjusted capitation rate, $p_{1}$ and $q_{1}$ are now the price and quantity of services included in the capitation basket, $p_{2}$ and 


\section{J. KANTAREVIC AND B. KRALJ}

$q_{2}$ are the price and quantity of services outside the basket, $z$ is the hard cap on the basket services provided to non-enrolled patients and, as before, $m$ is the number of enrolled patients and $n$ is the number of non-enrolled patients. To estimate this hypothetical base compensation in the FHO model, we used the actual profile of services provided to each patient in the fiscal year 2006 and the list of enrolled patients as of April 1, 2006. For $R$, we used the base rate of C $\$ 144.08$ multiplied by the age- and sex-specific modifier for each enrolled patient. These modifiers include 19 five-year age categories for each sex and range from 0.44 for men 10 to 14 years of age to 2.71 for women older than 90 years, with the provincial average standardized to 1 . To identify $q_{1}$ and $q_{2}$, we used the list of more than 100 fee codes specified in the FHO contract. Lastly, for $z$ we used the actual value of $\mathrm{C} \$ 47,500$ that applied in the fiscal year 2006.

\section{ACKNOWLEDGEMENTS}

We thank the editor, two anonymous referees, and seminar participants at McMaster University and at 2012 Workshop on Research Design for Causal Inference at Northwestern University.

\section{REFERENCES}

Abadie A, Imbens GW. 2008. On the failure of the bootstrap for matching estimators. Econometrica 76: 1537-1557.

Andrews DWK, Buchinsky M. 2000. A three-step method for choosing the number of bootstrap repetitions. Econometrica 67: 23-51.

Andrews DWK, Buchinsky M. 2001. Evaluation of a three-step method for choosing the number of bootstrap repetitions. Journal of Econometrics 103: 345-386.

Armour B, Pitts M, Maclean R, et al. 2001. The effect of explicit financial incentives on physician behavior. Archives of Internal Medicine 161: 1261-1266.

Becker S, Ichino A. 2002. Estimation of average treatment effects based on propensity scores. The Stata Journal 2(4): 358-377.

Blundell R, Costa Dias M. 2009. Alternative Approaches to Evaluation in Empirical Microeconomics. Journal of Human Resources 44(3): 565-640.

Canadian Diabetes Association. 2008. Clinical Practice Guidelines for the Prevention and Management of Diabetes in Canada. Canadian Journal of Diabetes 32(1): S1-S2010.

Christianson JB, Leatherman S, Sutherland K. 2008. Lessons from evaluations of purchaser pay-for-performance programs: A Review of the Evidence. Medical Care Research and Review 65(6): 5S-35.

Dali TM, Zhang Y, Chen YJ, Quick WW, Yang WG, Fogli J. 2010. The economic burden of diabetes. Health Affairs 29: $1-7$.

Dehejia RH, Wahba S. 2002. Propensity-score matching methods for nonexperimental causal studies. The Review of Economics and Statistics 84(1): 151-161.

Dusheiko M, Gravelle H, Martin S, Rice N, Smith PC. 2011. Does disease management in primary care reduce hospital costs? Evidence from English primary care. Journal of Health Economics 2:30(5): 919-32.

Eggleston K. 2005. Multitasking and mixed systems for provider payment. Journal of Health Economics 24: $211-223$.

Fan J. 1992. Design adaptive nonparametric regression. Journal of the American Statistical Association 87: 998-1004.

Fan J. 1993. Local linear regression smoothers and their minimax efficiencies. The Annals of Statistics 21: $196-216$.

Frolich A, Talavera JA, Broadhead P, Dudley RA. 2007. A behavioral model of clinician responses to incentives to improve quality. Health Policy 80(1): 179-193.

Glazier RH, Klein-Geltnik J, Kopp A, Sibley LM. 2009. Capitation and enhanced fee-for-service models for primary care reform: a population-based evaluation. Canadian Medical Association Journal 180(11): E72-E81.

Ham JC, Li X, Reagan PB. 2011. Matching and semiparametric IV estimation, a distance-based measure of migration, and the wages of young men. Journal of Econometrics 161(2): 208-227.

Heckman J, Hotz J. 1989. Choosing among alternative nonexperimental methods for estimating the impact of social programs: the case of manpower training. Journal of the American Statistical Association 84(408): 862-880.

Heckman J, Ichimura H, Todd P. 1997. Matching as an Econometric Evaluation Estimator: Evidence from Evaluating a Job Training Program. The Review of Economic Studies 64(4): 605-654.

Heckman J, Ichimura H, Todd P. 1998. Matching as an econometric evaluation estimator. The Review of Economic Studies 65(2): 261-294. 
Heckman J, Lozano S. 2004. Using matching, instrumental variables and control functions to estimate economic choice models. The Review of Economics and Statistics 86(1): 30-57.

Holmstrom B, Milgrom P. 1991. Multitask principal-agent analyses: incentive contracts, job design. Journal of Law, Economics and Organization 7(special issue): 24-52.

Institute for Clinical Evaluative Studies. 2003. Diabetes in Ontario - an ICES practice atlas. Available at: www.ices.on.ca/ file/DM_Intro.pdf.

Imbens GW, Wooldridge JM. 2009. Recent developments in the econometrics of program evaluation. Journal of Economic Literature 47(1): 5-86.

Integrated Healthcare Association. 2006. Advancing quality through collaboration: The California pay for performance program. www.iha.org/pdfs_documents/p4p_california/P4PWhitePaper1_February2009.pdf.

International Diabetes Federation. 2010. Annual report. Available at: www.idf.org/sites/default/files/Annual-Report-2010FINAL-EN_0.pdf.

Kaarboe O, Siciliani L. 2011. Multi-tasking, quality and pay for performance. Health Economics 20: $225-238$.

Kantarevic J, Kralj B, Weinkauf D. 2011. Enhanced Fee-for-Service Model and Physician Productivity: Evidence from Family Health Groups in Ontario. Journal of Health Economics 30(1): 99-111.

Kantarevic J, Kralj B. 2013. Quality and Quantity in Primary Care Mixed Payment Models: Evidence from Family Health Organizations in Ontario. Canadian Journal of Economics 46(1).

Léger PT. 2008. Physician Payment Mechanisms. In: Financing Health Care: New Ideas for a Changing Society, Lu M, Jonsson E (eds.), Wiley: Wiley-VCH Verlag GmbH \& Co. KGaA; 149-176.

Leuven E, Sianesi B. 2003. PSMATCH2: Stata module to perform full Mahalanobis and propensity score matching, common support graphing, and covariate imbalance testing. http://ideas.repec.org/c/boc/bocode/s432001.html. Version 3.1.5.

Li J, Hurley J, DeCicca P, Buckley G. 2011. Physician response to pay-for-performance - evidence from a natural experiment. NBER Working Paper 16909.

Lindenauer P, Remus D, Roman S, Rothberg MB, Benjamin EM, Ma A, Bratzler DW. 2007. Public reporting and pay for performance in hospital quality improvement. The New England Journal of Medicine 356(5): 486-496.

McGuire TG. 2000. Physician agency. In: Culyer AJ, Newhouse JP (eds.), Handbook of Health Economics, vol. 1A. NorthHolland, Amsterdam; 461-536.

Ontario Ministry of Health and Long Term Care, Schedule of Benefits, Physician Services under the Health Insurance Act (September 1, 2011).

Petersen L, Woodard L, Urech T, Daw C, Sookanan S. 2006. Does pay-for-performance improve the quality of health care? Annals of Internal Medicine 145(4): 265-272

Rosenbaum PR, Rubin DB. 1983. The central role of the propensity score in observational studies for causal effects. Biometrika 70(1): 41-55.

Rosenbaum PR, Rubin DB. 1985. Constructing a control group using multivariate matched sampling methods that incorporate the propensity score. The American Statistician 39(1): 33-38.

Rosenthal MB, Frank RG. 2006. What is the empirical basis for paying for quality in health care? Medical Care Research and Review 63(2): 135-157.

Rosenthal MB, Frank RG, Li Z, Epstein AM. 2005. Early experience with pay-for-performance: from concept to practice. Journal of the American Medical Association 294(14): 1788-1793.

Silverman B. 1986. Density Estimation for Statistics and Data Analysis. Chapman \& Hall: London.

Smith PC, York N. 2004. Quality incentives: the case of U.K. general practitioners. Health Affairs 23(3): 112-118.

Smith J, Todd P. 2005. Does matching overcome LaLonde's critique of nonexperimental estimators? Journal of Econometrics 125: 305-353.

Town R, Kane R, Johnson P, Butler M. 2005. Economic incentives and physicians' delivery of preventive care: a systematic review. American Journal of Preventive Medicine 28(2): 234-240. 